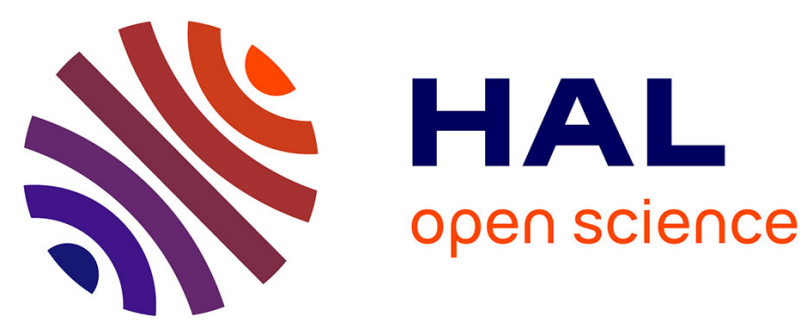

\title{
COXINEL transport of laser plasma accelerated electrons
}

Driss Oumbarek Espinos, Amin Ghaith, Alexandre Loulergue, Thomas André, Charles Kitégi, Mourad Sebdaoui, Fabrice Marteau, Frédéric Blache, Mathieu Valléau, Marie Labat, et al.

\section{To cite this version:}

Driss Oumbarek Espinos, Amin Ghaith, Alexandre Loulergue, Thomas André, Charles Kitégi, et al.. COXINEL transport of laser plasma accelerated electrons. Plasma Phys.Control.Fusion, 2020, 62 (3), pp.034001. 10.1088/1361-6587/ab5fec . hal-03090346

\section{HAL Id: hal-03090346 \\ https://hal.science/hal-03090346}

Submitted on 2 Jan 2021

HAL is a multi-disciplinary open access archive for the deposit and dissemination of scientific research documents, whether they are published or not. The documents may come from teaching and research institutions in France or abroad, or from public or private research centers.
L'archive ouverte pluridisciplinaire HAL, est destinée au dépôt et à la diffusion de documents scientifiques de niveau recherche, publiés ou non, émanant des établissements d'enseignement et de recherche français ou étrangers, des laboratoires publics ou privés. 


\title{
COXINEL transport of laser plasma accelerated electrons
}

\author{
Driss Oumbarek Espinos ${ }^{1,2,3}$, Amin Ghaith ${ }^{1,3}$, Alexandre \\ Loulergue ${ }^{1}$, Thomas André ${ }^{1}$, Charles Kitégi ${ }^{1}$, \\ Mourad Sebdaoui ${ }^{1}$, Fabrice Marteau ${ }^{1}$, Frédéric Blache ${ }^{1}$, \\ Mathieu Valléau ${ }^{1}$, Marie Labat ${ }^{1}$, Alain Lestrade ${ }^{1}$, Eléonore \\ Roussel ${ }^{4}$, Cédric Thaury ${ }^{5}$, Sébastien Corde ${ }^{5}$, Guillaume \\ Lambert ${ }^{5}$, Olena Kononenko ${ }^{5}$, Jean-Philippe Goddet ${ }^{5}$, \\ Amar Tafzi ${ }^{5}$, Igor Andriyash ${ }^{6}$, Victor Malka ${ }^{5,6}$ and \\ Marie-Emmanuelle Couprie ${ }^{1,}$ \\ ${ }^{1}$ Synchrotron SOLEIL, Saint-Aubin, Gif-sur-Yvette, 91192, France \\ ${ }^{2}$ Graduate School of Engineering, Osaka University, 2-8 Yamadaoka, Suita, Osaka \\ 565-0871, Japan \\ ${ }^{3}$ Université Paris-Saclay, Paris 91190, France \\ ${ }^{4}$ Univ. Lille, CNRS, UMR 8523 - PhLAM - Physique des Lasers Atomes et \\ Molécules, F-59000 Lille, France \\ ${ }^{5}$ LOA, ENSTA Paris, CNRS, Ecole Polytechnique, Institut Polytechnique de Paris, \\ 828 Boulevard des Maréchaux, 91762 Palaiseau, France \\ ${ }^{6}$ Department of Physics of Complex Systems, Weizmann Institute of Science, \\ Rehovot 761001, Israel \\ E-mail: driss.oumbarek-espinos@synchrotron-soleil.fr
}

\begin{abstract}
Laser Plasma Acceleration (LPA) enables to generate up to several GeV electron beam with short bunch length and high peak current within centimeters scale. In view of undulator type light source applications, an electron beam manipulation has to be applied. We report here on detailed electron beam transport for an LPA electron beam on the COXINEL test line, that consists of strong permanent quadrupoles to handle the electron beam divergence, a magnetic chicane to reduce the energy spread and a second set of quadrupoles for adjusting the focusing inside the undulator. After describing the measured LPA characteristics, we show that we can properly transport the electron beam along the line, thanks to several screens. We also illustrate the influence of the chromatic effects induced by the electron beam energy spread, both experimentally and numerically. We then study the sensitivity of the transport to the electron beam pointing and skew quadrupolar components.
\end{abstract}

Keywords: Laser Plasma Acceleration, Electron beam transport, Quadrupole, Free electron laser

Submitted to: Plasma Phys. Control. Fusion 


\section{Introduction}

In Laser Plasma Acceleration (LPA) [1], an ultra short laser pulse generates a plasma wave with relativistic phase velocity via the ponderomotive force. Particles can be accelerated, with electric fields up to three orders of magnitude larger $(>100 \mathrm{GeV} / \mathrm{m})$ [2] than conventional radio frequency accelerators, in a range covering from tens of $\mathrm{MeV}$ to GeVs [3, 4] with fs duration [5], excellent emittance at the plasma-vacuum interface ( 1 mm.mrad), high peak current (1-10 kA) and percent energy spread [6] within $\mathrm{cm}$ scale. Nevertheless, the best LPA parameters are not achieved simultaneously. Different configurations (laser wakefield accelerator (LWFA) [1], plasma beat wave accelerator (PBWA) [1], self-modulated LWFA [7], wakefields driven by multiple pulses [2] and the highly nonlinear regime of electron cavitation [8]) offer distinct characteristics. In the generation of the electron bunch by LPA, two phases can be differentiated, injection, during which the electrons are injected into the wave forming the beam, and boost where the electrons of the beam gain energy. The method used for injecting the electrons in the bubble (colliding pulse regime [9, 10], density ramp injection [11], ionization injection $[12,13,14])$ determines the characteristics of the LPA system.

The improvement of LPA motivated the development of shorter accelerators for high energy physics [15] and light source applications [16, 17, 18, 19, 20, 21, 22]. Many challenges still need to be overcome in order to achieve a stable and high performance system. The beams with high divergence and percentage level energy spread are difficult to capture and transport for applications due to fast emittance worsening [23, 24, 25]. Indeed, the geometrical emittance $\epsilon_{g}$ evolution is strongly dominated by the initial divergence $\sigma_{i}^{\prime}$, the energy spread $\sigma_{\gamma}$ and the roll angle of the quadrupoles $\theta$ as:

$$
\epsilon_{g, x}^{2} \approx \epsilon_{g, x, i}^{2}+4 \theta^{2} \sigma_{x, i}^{\prime 2} \sigma_{z, i}^{\prime 2} L^{2}+L^{2} \sigma_{x, i}^{\prime 4} \sigma_{\gamma}^{2}
$$

with $\mathrm{L}$ the drift distance $[23,26,27]$. Thus, for a current $I$, the peak brightness, defined as [28]:

$$
B=\frac{2 \times I}{\pi^{2} \epsilon_{g, z} \epsilon_{g, x}}
$$

used as beam quality figure of merit, also deteriorates. In addition, to achieve free electron laser (FEL) amplification the beam relative energy spread $\sigma_{\gamma}[29]$ should be smaller than the Pierce parameter [30]. The width of the electron energy slice should be inferior to the natural bandwidth of the first harmonic [29]. Hence, specific techniques to effectively transport and control the electron beam, while avoiding a decrease in quality and improving some of its characteristics, are required [31, 32, 33].

Studies about LPA beam dynamics in a transport line are carried out in the COXINEL line (COherent Xray source INferred from Electrons accelerated by Laser), which has been conceived with a final purpose of demonstrating FEL amplification using LPA $[34,35]$. The Pierce parameter condition on $\sigma_{\gamma}$ translates for the COXINEL undulator (107 periods of $18.16 \mathrm{~mm}$ [36]) to $176 \pm 0.5 \% \mathrm{MeV}$. The COXINEL line has already achieved electron beam transport from the source to an undulator while properly 
Table 1. Baseline parameters at the source of the COXINEL line design, beam characteristics at the undulator center after transport for a flat-top initial beam with the "undulator slit" optics and the slit opened at $3.6 \mathrm{~mm}$ and $1 \mathrm{~mm}$.

\begin{tabular}{|c|c|c|c|c|c|c|c|c|c|c|c|}
\hline Position & Slit & $\begin{array}{c}\text { Total } \\
\text { charge } \\
Q_{t}\end{array}$ & $\begin{array}{c}176 \pm 0.5 \mathrm{MeV} \\
\text { slice charge } \\
Q_{176 \pm 0.5 \mathrm{MeV}}\end{array}$ & $\begin{array}{c}\text { Energy } \\
\text { spread } \\
\sigma_{\gamma}\end{array}$ & $\begin{array}{c}\text { Divergence } \\
\sigma_{x}^{\prime}, \sigma_{z}^{\prime}\end{array}$ & $\begin{array}{c}\text { Emittance } \mathrm{x} \\
\epsilon_{x}\end{array}$ & $\begin{array}{c}\text { Emittance } \mathbf{z} \\
\epsilon_{z}\end{array}$ & $\begin{array}{c}\text { Beam } \\
\text { length } \\
\sigma_{s}\end{array}$ & $\begin{array}{c}\text { Beam } \\
\text { size } \mathrm{x} \\
\sigma_{x}\end{array}$ & $\begin{array}{c}\text { Beam } \\
\text { size } \mathbf{z} \\
\sigma_{z}\end{array}$ & $\begin{array}{r}\text { Central } \\
\text { energy }\end{array}$ \\
\hline & $m m$ & $p C$ & $p C$ & & mrad & mm.mrad & mm.mrad & $\mu m$ & $\mathrm{~mm}$ & $\mathrm{~mm}$ & $\mathrm{MeV}$ \\
\hline Source & - & 34 & - & $1 \%$ & 1 & 1 & 1 & 1 & - & - & 176 \\
\hline Undulator center & 3.6 & 34 & 10.9 & $1 \%$ & - & 1.7 & 1.4 & - & 0.05 & 0.03 & - \\
\hline Undulator center & 1 & 28.5 & 3.3 & $0.8 \%$ & - & 1.6 & 1.3 & - & 0.05 & 0.03 & - \\
\hline
\end{tabular}

focusing the beam and adapting to pointing instabilities [37]. In this paper, a study of LPA beam behavior through a transport line is carried. The adaptability of the COXINEL line is shown and the typical beam characteristics obtained with an LPA source based on the ionization injection scheme are analyzed. Then, the transport through an ideal line with measured initial electron beam is examined and compared with experiment. The effects of a non-optimized gradient of the quadrupole triplet is explored for ideal cases and experiment. The sensitivity of the beam characteristics to pointing at the source is shown. Finally, the effect of a roll angle in the quadrupole triplet is reproduced via simulations and compared to experiment. The degradation suffered by the total beam and also the slice of interest, quantified by the brightness, is shown through experiment and simulations.

\section{COXINEL transport line}

The COXINEL line (see Figure 1) aims at demonstrating FEL capabilities from an LPA based electron beam [38, 39, 34, 40] and is designed for the baseline parameters in Table 1. The beam is transported from the source to the center of the undulator while being modified to fulfill the FEL requirements. The LPA system, generating the electron bunch, is composed of a Titanium:Sapphire (Ti:Sa) laser and a gas jet. $5 \mathrm{~cm}$ after the source, a triplet of variable gradient quadrupoles called QUAPEVAs [41, 42] handles the beam via a strong gradient $[21,43,44]$. Then, a magnetic chicane composed of four dipole magnets sorts the electrons by energy in the horizontal plane. To reduce the energy spread $\sigma_{\gamma}$, a removable slit, located at the chicane center, selects a small energy range around an energy of interest $[24,37,45,46,47]$. Thus, the slit reduces $\sigma_{\gamma}$ at the expenses of the total beam charge. Finally, a set of four electromagnetic quadrupoles (EMQs) (up to $20 \mathrm{~T} / \mathrm{m}$ ) focuses the beam at the center of the undulator.

The line counts with multiple beam diagnostics. A removable electron spectrometer, that can be introduced at the position of the QUAPEVA triplet, permits to estimate the initial electron beam parameters. Before and after the undulator, two integrating current transformers (ICT) are used to measure the total beam charge. Five removable imagers are positioned after the QUAPEVA triplet, at the chicane center, undulator entrance and exit and after the dipole dump, allowing for electron beam observation and total 
charge measurements at the undulator exit and the electron dump after calibration via an ICT.

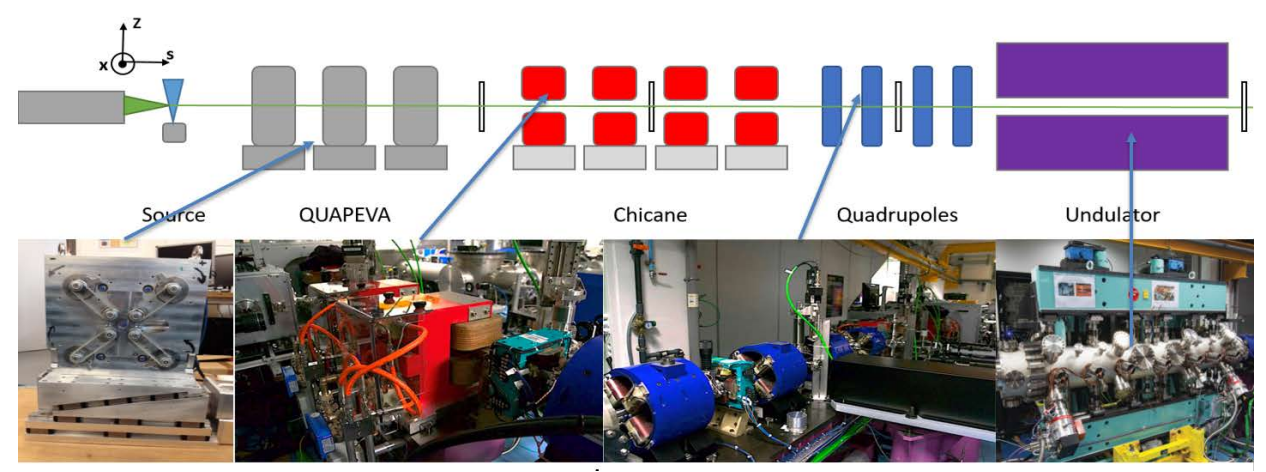

Figure 1. COXINEL transport line scheme. Left to right: Laser pulse focused on a gas jet (blue), triplet of QUAPEVAs (grey), first screen for electron beam profile measurement (white), chicane (red), a second set of electro-magnetic quadrupoles (blue), screen before and after the undulator (black) and cryo-ready undulator (purple).

\subsection{COXINEL optics}

For the electron beam transport simulations, the line is modeled up to second order (BETA [48]) and the electrons are tracked in a 6D phase space (positions, momenta, energy) via a $6 \mathrm{D}$ tracking pass code from the source to the undulator dipole dump [39]. The code has been benchmarked with ASTRA [49, 34], ELEGANT [50] and OCELOT [51]. The adaptability of the line components gives the ability to change to different configurations in order to check the beam positioning and properties at different locations of the line and to correct any misalignments.

Figure 2a shows the horizontal and vertical beam envelopes along the entire line in the case of a flat-top $176 \pm 0.01 \mathrm{MeV}$ beam for different COXINEL optics configurations and Table 2 presents the corresponding magnetic lattice characteristics for each optics. The different optics focus the beam in both horizontal and vertical planes at different positions along the line. The "slit" optics focuses the beam horizontally at the center of the chicane to select the energy of the electron beam. Additionally, the electron beam can be refocused in the undulator center with the "undulator slit optics". Three other optics can be used to directly image the electron beam at the undulator entrance, center and exit ("undulator entrance, center and exit optics"). Figure 2b shows the horizontal and vertical beam envelope along the entire line for the "undulator slit" optics for a beam with the $176 \mathrm{MeV}$ reference energy and $\sigma_{\gamma}=5 \%, 1 \%, 0.01 \%$. When the $\sigma_{\gamma}$ increases, the focusing in the undulator center degradates.

Figure 3 shows the transport along the line for a beam with the baseline parameters (Table 1) and the "undulator slit" optics with the slit opened at $3.6 \mathrm{~mm}$. Table 1 shows the beam parameters at the undulator center. For a beam of $1 \%$ energy spread at the reference energy, a small increase of the emittance occurs while the charge is conserved 


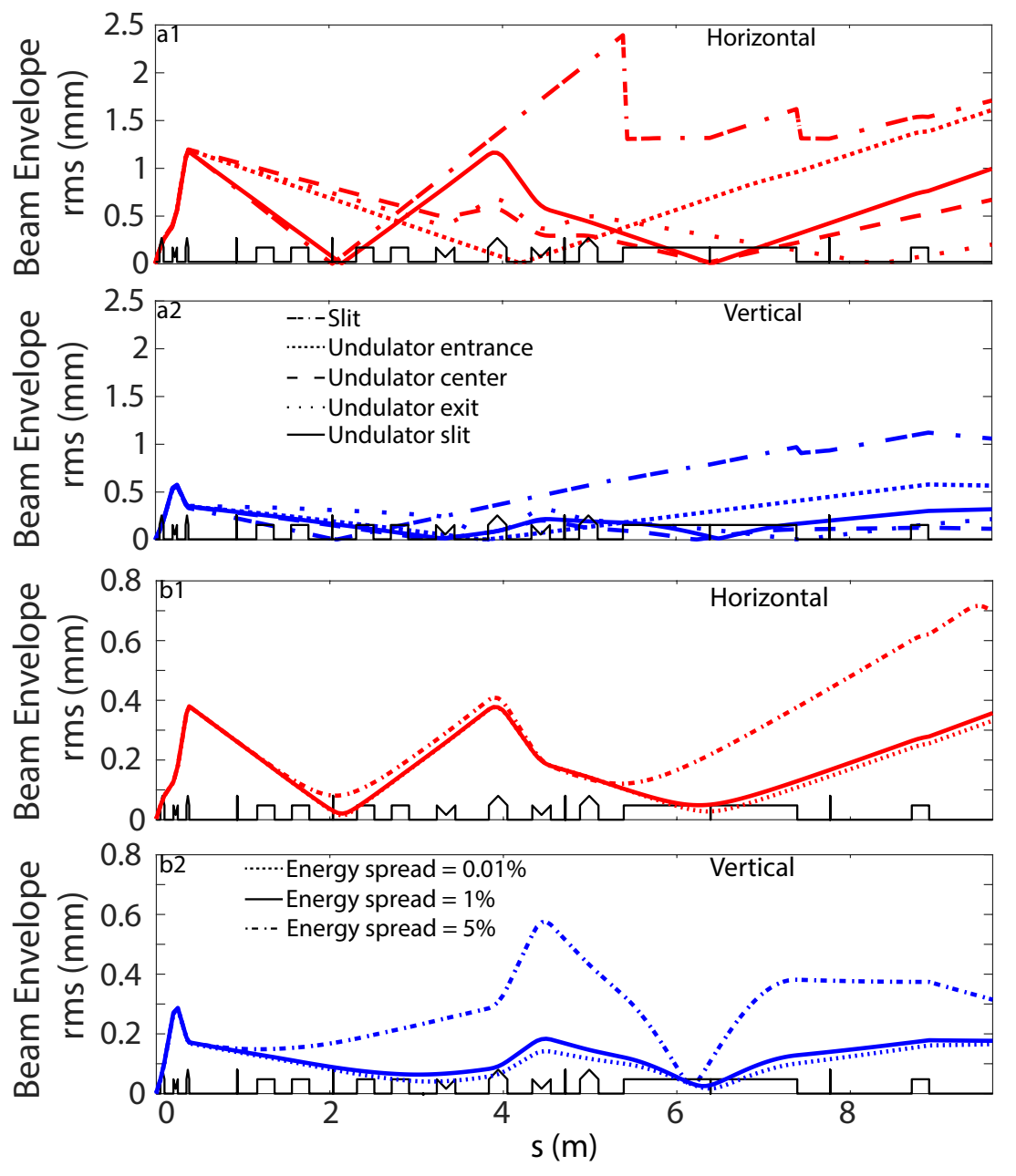

Figure 2. Simulation of the horizontal (red) and vertical (blue) beam envelopes versus longitudinal position "s" of (a1-2) the optics "slit", "undulator entrance", "undulator center", "undulator exit" and "undulator slit" along the transport line for a flat-top energy spectrum of $176 \pm 0.01 \mathrm{MeV}$ and (b1-2) the optics "undulator slit" along the transport line for a flat-top energy spectrum of $176 \mathrm{MeV}$ with $\sigma_{\gamma}=5 \%, 1 \%$, $0.01 \%$. The beam parameters are $\epsilon_{x, i}, \epsilon_{z, i}=0.2 \mathrm{~mm} . \mathrm{mrad}, \sigma_{z, i}^{\prime}=2 \mathrm{mrad}, \sigma_{x, i}^{\prime}=3.12$ mrad RMS. Transport line elements representation (black) with screens (vertical line), dipoles (rectangle), focusing (rectangle with indentation) and defocusing quadrupoles (triangle on top).

at the undulator center and the beam is properly focused. For a flat-top beam of $10 \%$ energy spread around the reference energy $176 \mathrm{MeV}$, for the slit opened at $3.6 \mathrm{~mm}$ (1 $\mathrm{mm})$ the $\sigma_{\gamma}$ is reduced to $5.5 \%(0.8 \%)$ (Table 1$)$.

\section{LPA electron beam characteristics}

The COXINEL line uses the laser system at "Salle Jaune" of Laboratoire d'Optique Appliquée for the LPA source. The Ti:Sa laser provides a linearly polarized light on the horizontal direction, $30 \mathrm{fs}$ (FWHM), $60 \mathrm{TW}, 800 \mathrm{~nm}$ pulse focused in a $20 \mu \mathrm{m}$ spot into 
Table 2. Magnetic elements characteristics per optics configuration. The chicane dipoles always with a gap of $25 \mathrm{~mm}$, magnetic field $0.24 \mathrm{~T}$ with a current of $46.5 \mathrm{~A}$ and chicane dispersion $\left(r_{56}\right)$ of $4.3 \mathrm{~mm}$.

\begin{tabular}{|c|c|c|c|c|c|c|c|}
\hline Optics & \multicolumn{3}{|c|}{$\begin{array}{l}\text { QUAPEVAs } \\
\text { (QAP) }\end{array}$} & \multicolumn{4}{|c|}{$\begin{array}{c}\text { Electro-Magnetic } \\
\text { Quadrupoles (EMQ) }\end{array}$} \\
\hline Unit & \multicolumn{3}{|c|}{$\begin{array}{c}\text { Gradient } \\
\mathrm{T} / \mathrm{m}\end{array}$} & \multicolumn{4}{|c|}{$\begin{array}{l}\text { Gradient } \\
\mathrm{T} / \mathrm{m}\end{array}$} \\
\hline Component & QAP1 & QAP2 & QAP3 & EMQ1 & EMQ2 & EMQ3 & EMQ4 \\
\hline "Slit" & +104.8 & -104.3 & +97.36 & 0 & 0 & 0 & 0 \\
\hline "Undulator entrance" & +102.8 & -101.2 & +90.26 & 0 & 0 & 0 & 0 \\
\hline "Undulator" & +102.68 & -101.14 & +89.10 & -0.52 & 0.85 & -1.23 & 0.46 \\
\hline "Undulator exit" & +102.41 & -100.74 & +89.78 & -1.74 & 1.26 & -1.36 & 0.41 \\
\hline "Undulator slit" & +104.1 & -103 & +96.43 & -0.01 & 4.70 & -4.40 & 0.29 \\
\hline
\end{tabular}
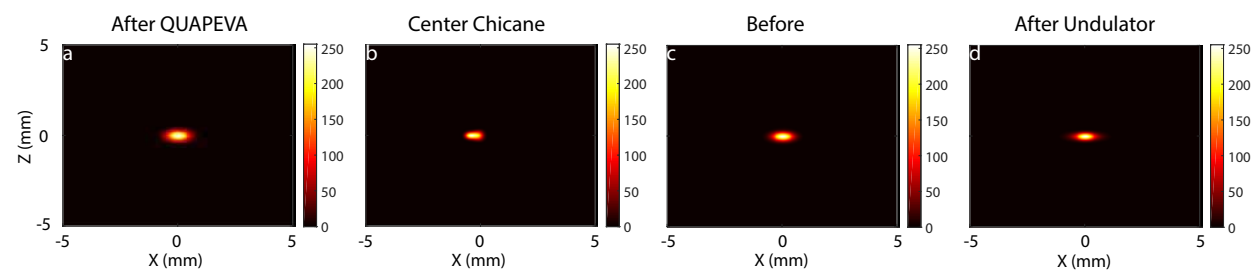

Figure 3. Electron beam transverse shape simulated after the QUAPEVA triplet (a), center of the chicane (b), undulator entrance (c) and exit (d) for a flat-top beam centered at $176 \mathrm{MeV}$ with the baseline parameters (Table 1) with the "undulator slit" optics.

the supersonic gas jet with a millimeter scale nozzle, using a gas mixture of $99 \%$ Helium and $1 \%$ Nitrogen. The LPA system is operated in the bubble regime with ionization injection $[12,13,14]$, using a medium of low-Z gas with a small proportion of high-Z gas: the ionized outer-shell electrons of the high-Z gas contribute mainly to the plasma wave and the inner-shell ionized electrons of the high-Z are more easily trapped [12]. This configuration is robust and capable of producing beams of relatively high charge.

The electron beam energy distribution and the vertical divergence are measured with the removable spectrometer placed $355 \mathrm{~mm}$ from the source and is composed of a 100-mm long permanent dipole magnet of $1.1 \mathrm{~T}$. The electron beam is dispersed horizontally and imaged on a lanex scintillating screen with a CCD camera. The resolution of the spectrometer spans from $2.7 \%$ up to $3.8 \%$ for energies between 50 and $350 \mathrm{MeV}$. A removable imager positioned $64 \mathrm{~cm}$ from the source indirectly measure an approximation of the horizontal divergence of the beam. The ratio $r=\frac{\sigma_{x}}{\sigma_{z}}$ between the horizontal beam size $\sigma_{x}$ and the vertical beam size $\sigma_{z}$ after a drift is assumed to be equal to the ratio between the horizontal divergence $\sigma_{x}^{\prime}$ and the vertical beam divergence 
Table 3. Typical distribution energy range $E_{\text {range }}$, average total charge $Q_{t, i}$, slice $176 \pm 0.5 \mathrm{MeV}$ charge $Q_{i, 176 \pm 0.5}$, vertical divergence $\sigma_{z, i, 176 \pm 0.5}^{\prime}$, ratio r, emittance $\epsilon_{x, i}, \epsilon_{z, i}$ and beam length $\sigma_{s, i}$ obtained at the LPA source (Figure 4a,b,c). $\epsilon_{x, i}, \epsilon_{z, i}$ and $\sigma_{s, i}$ obtained through particle in cell simulations (PIC)

\begin{tabular}{ccccccc}
\hline$E_{\text {range }}$ & $Q_{t, i}$ & $Q_{i, 176 \pm 0.5}$ & $\sigma_{z, i, 176 \pm 0.5}^{\prime}$ & $\mathbf{r}$ & $\epsilon_{x, i}, \epsilon_{z, i}$ & $\sigma_{s, i}$ \\
\hline$M e V$ & $p C$ & $p C$ & $m r a d$ & & mm.mrad & $\mu m$ \\
\hline $50-300$ & $148 \pm 95$ & $0.01-0.5$ & $1.5-5$ & $1.5-3$ & 0.2 & 1 \\
\hline
\end{tabular}

Table 4. Total charge, slice $176 \pm 0.5 \mathrm{MeV}$ charge and vertical divergence of the initial distributions of Figure 5.

\begin{tabular}{ccccc}
\hline Distribution & Shots & $Q_{t}$ & $Q_{176 \pm 0.5}$ & $\sigma_{z, 176 \pm 0.5}^{\prime}$ \\
\hline & & $p C$ & $p C$ & mrad \\
\hline Experimental campaign (Figure 5a) & 370 & 196 & 0.3 & 1.9 \\
Good experimental campaign data (Figure 5b) & 160 & 215 & 0.5 & 2 \\
Set average (Figure 5c) & 20 & 245 & 0.22 & 2.2 \\
Single-shot (Figure 5d) & 1 & 239 & 0.51 & 2.1 \\
\hline
\end{tabular}

$\sigma_{z}^{\prime}$ at the LPA source. Figure 4a,b present the electron distribution and the total charge evolution at the spectrometer during one experimental campaign (739 shots). Table 3 shows the range of values obtained from the LPA source. After the energy selection of the slice of interest with the chicane slit and the aperture of the line, the total charge drops (Figure 4d). Experimental LPA parameters significantly differs from the baseline ones (see Table 3, Table 1). Consequently, the adaptability of the transport line is key to work with an LPA source.

Figure 5 shows the measured energy distribution and divergence (see Table 4) during an experimental campaign, with the average (a), 43\% best shots (b), 20 shot series (c) and a single shot case from a previous campaign (d). As seen in Figure 4a, the beam distribution changes significantly from day to day and even in the same day. The distribution resulting from an average of a set (20 consecutive measured beams) (Figure 5c, Table 4) presents a ratio $\mathrm{r}=1.56$ and a shot-to-shot pointing variation on the first beam imager for the data set of $2.62 \mathrm{mrad}$ (RMS) in the horizontal direction and $0.49 \mathrm{mrad}$ (RMS) in the vertical direction. Figure 5d (Table 4) presents a single shot distribution from an older experimental campaign. 

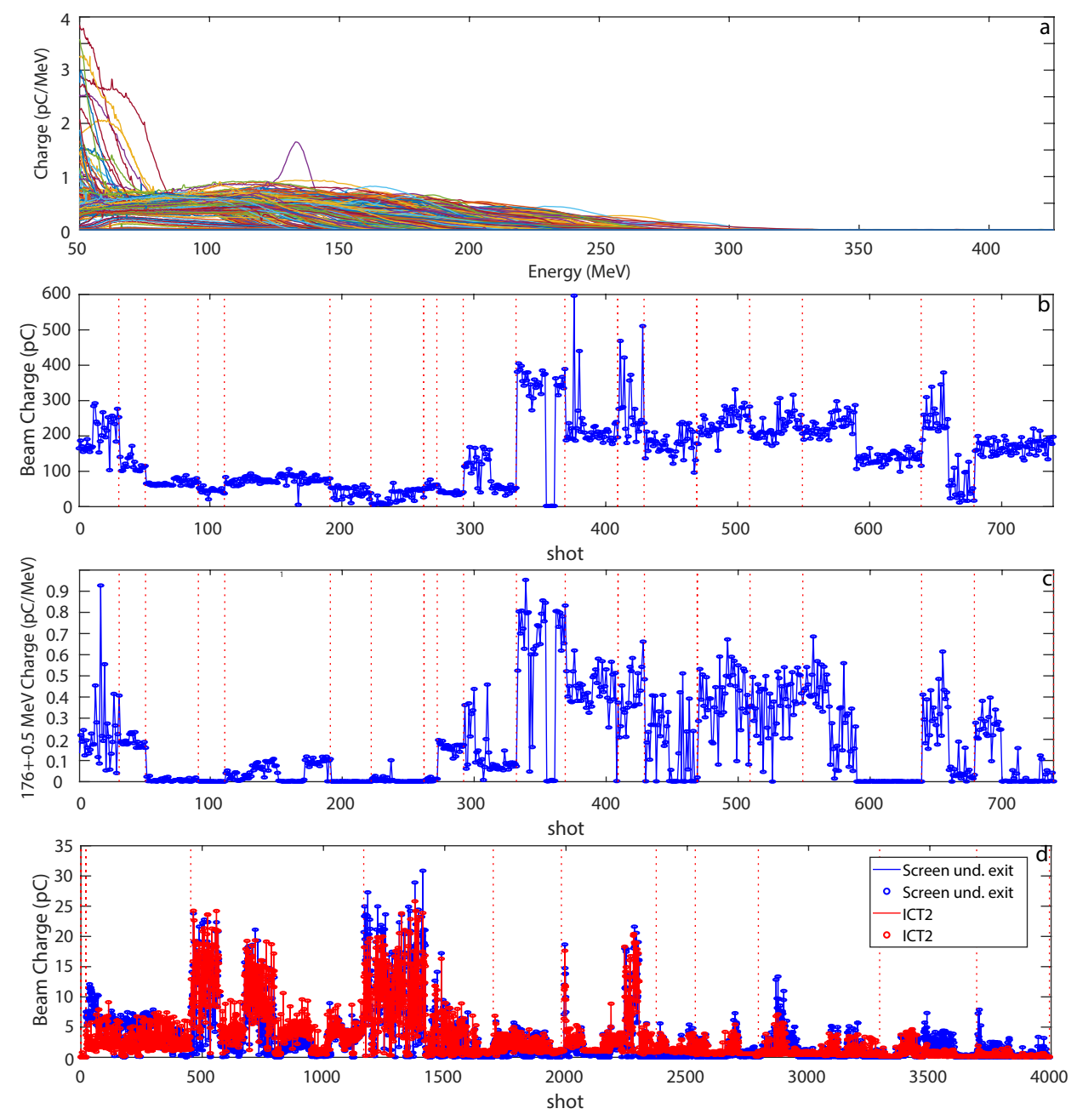

Figure 4. Electron beam characterization at the source. (a) Electron distributions, (b) total charge corresponding to 739 shots, (c) slice $176 \pm 0.5 \mathrm{MeV}$ charge corresponding to 739 shots measured using the electron spectrometer and (d) total charge at the undulator exit measured with an ICT (red) and by the camera (blue) during an entire experimental campaign separated by days, corresponding to 3993 shots (red dotted line).

\section{Electron beam transport}

\subsection{From the source to the beam dump}

Figure 6a-e shows a simulation of a transport from the source to the electron dump with the initial distribution of Figure $5 \mathrm{~b}$ in the case of perfect quadrupoles. While the total charge arriving at the beam dump is of only $13 \mathrm{pC}$, the $176 \pm 0.5 \mathrm{MeV}$ slice charge is roughly conserved $(0.5 \mathrm{pC}$ at the source and $0.49 \mathrm{pC}$ at dump). In the case of the measurements of an entire experimental campaign (Figure 6f), the average total charge measured at the undulator exit ICT is of $1 \mathrm{pC}$ (standard deviation (STD) 1.7 $\mathrm{pC}$ ) while at the beam dump screen is of $1.4 \mathrm{pC}$ (STD $1.9 \mathrm{pC})$. Both measurements and simulations present similar evolution. 

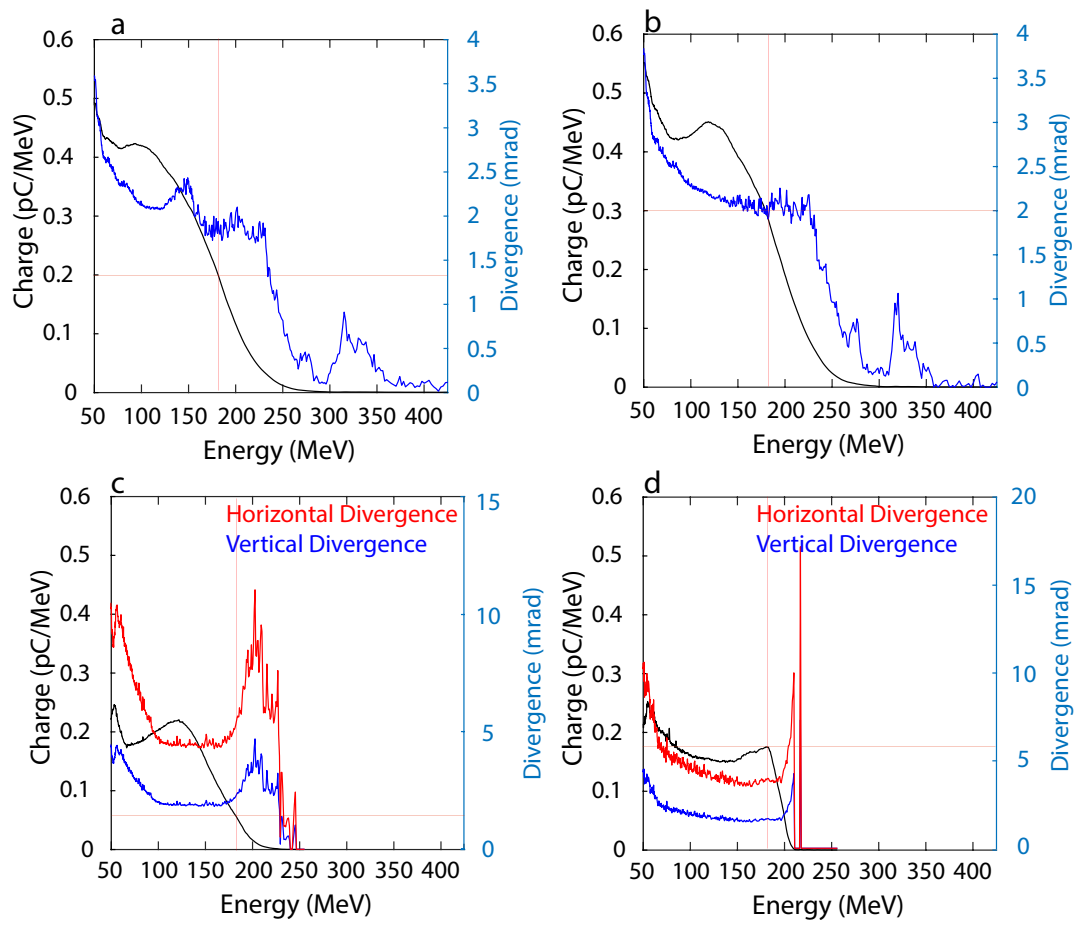

Figure 5. Initial beam energy distribution (black), vertical (blue), and horizontal (red) RMS divergence for energy slices of $\pm 0.3 \mathrm{MeV}$, spectrometer resolution. (a) Average of the experimental campaign, 370 shots (Figure 4a), (b) without the worst days, 160 shots, (c) Average over a set of 20 distributions and (d) one-shot of a previous experimental campaign.

\subsection{Beam chromatic effects during transport}

Let's consider the chromatic effects in the COXINEL case (Equation (1)). Figure 7 presents the effects of changing the gradient of the second QUAPEVA, the strongest one, on the beam at the undulator entrance with the "undulator entrance" optics. Figure 7ae1 shows the transverse electron beam distributions for the baseline beam (Table 1). For the reference gradient $0 \%$, the beam is properly focused at the center of the screen with a spot of $\sigma_{x}=0.04 \mathrm{~mm}$ and $\sigma_{z}=0.08 \mathrm{~mm}$ RMS. When the gradient is modified, the beam starts to elongate in the vertical direction achieving $\sigma_{x}=0.07 \mathrm{~mm}$ and $\sigma_{z}=0.45 \mathrm{~mm}$ RMS ( $\sigma_{x}=0.07 \mathrm{~mm}$ and $\sigma_{z}=0.41 \mathrm{~mm}$ RMS) for a gradient of $+3 \%(-3 \%)$. The modification of the optimized gradient causes the defocusing in the vertical direction of the electrons of energies different from $176 \mathrm{MeV}$. Figure $7 \mathrm{a}-\mathrm{e} 2$ presents the influence of the gradient for the average beam of Figure 5c. For the optimized gradient (reference case 0\%), the beam, in particular the $176 \pm 1 \mathrm{MeV}$ slice, is properly focused at the center of the screen surrounded by a halo from the further energies. A modification of $\pm 1 \%$ or more of the reference gradient immediately causes the apparition of a cross due to the horizontal and vertical focusing in different positions for each electron energy. Thus, when the gradient suffers a change of $1 \%(-1 \%)$ or higher (lower) the electrons of higher energies are focused vertically (horizontally) and the lower energies horizontally (vertically). The 

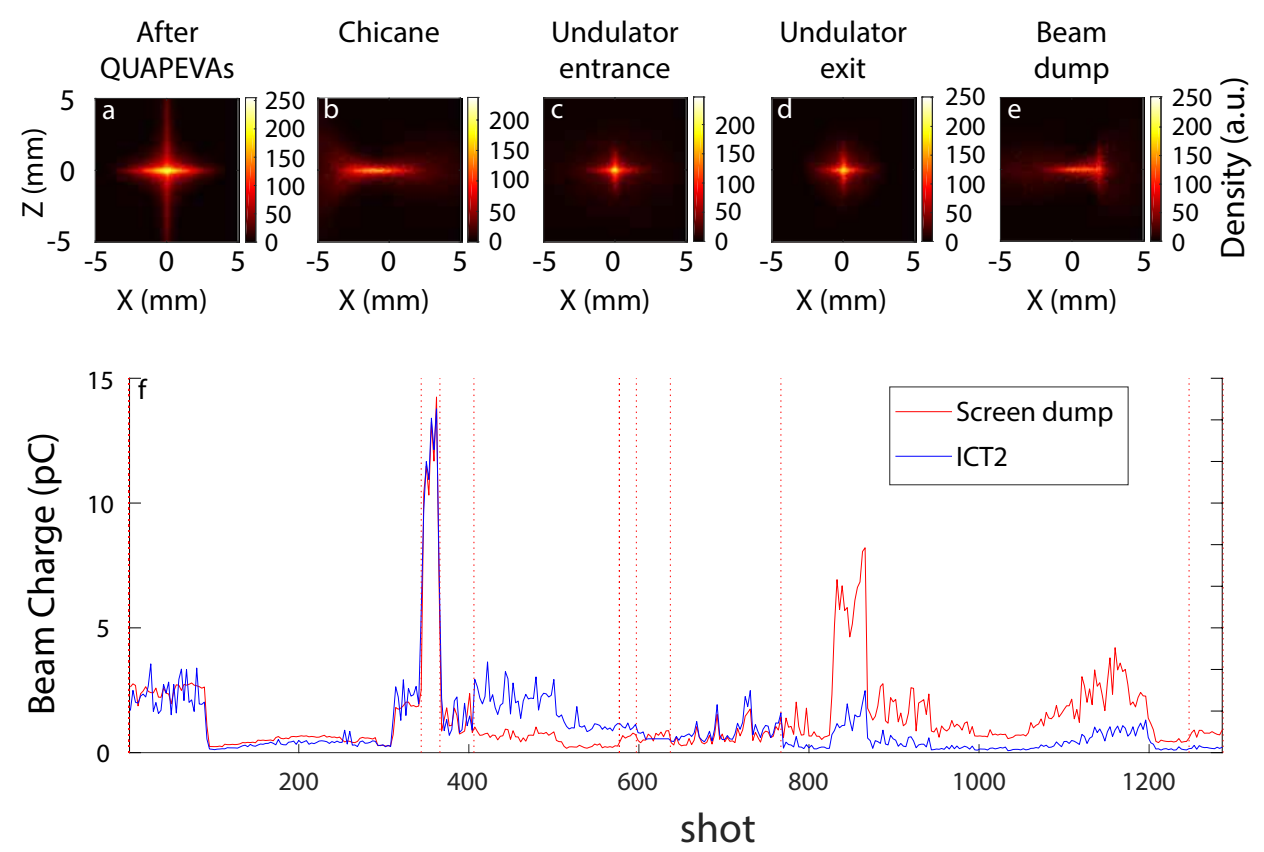

Figure 6. Electron beam transverse shape simulated (a) after the QUAPEVA triplet, (b) at center of the chicane, (c) at undulator entrance, (d) exit and (e) electron dump for the average beam of Figure $5 \mathrm{~b}, Q_{i}=100 \mathrm{pC}, \epsilon_{x, i}, \epsilon_{z, i}=0.2 \mathrm{~mm} . \mathrm{mrad}, \sigma_{z, i}^{\prime}=2 \mathrm{mrad}$, $\sigma_{x, i}^{\prime}=3.12 \mathrm{mrad}$ with the "undulator" optics. (f) Total beam charge measured with the ICT at the undulator exit (blue), at the electron dump screen (red) during an entire experimental campaign, 1287 shots, chronologically organized.

measurements (Figure 7a-e3) show a good agreement with their simulated counterparts. For the optimized gradient the measured beam has a horizontal beam size $\sigma_{x}=430 \mu \mathrm{m}$ and vertical size $\sigma_{z}=270 \mu \mathrm{m}$ while the simulated case has $\sigma_{x}=490 \mu \mathrm{m}$ and $\sigma_{z}=670 \mu \mathrm{m}$. The $\sigma_{z}$ difference between measurement and simulation is probably due to the inherent unknowns of the experimental parameters of each shot.

\section{Transport sensitivity}

\subsection{Beam pointing effect}

Figure 8 shows the transverse beam shape along the transport line with the "undulator slit" optics for the on axis beam (Figure 8a-d2) and for a $2 \mathrm{mrad}$ vertical off-axis pointing case (Figure 8a-d1). The vertical off-axis pointing substantially defocuses the beam in the same direction along the line as compared to the on axis case, especially in the middle of the chicane where the beam suffers from a tilt which potentially worsens the energy selection capabilities of the slit (Figure 8b1). Table 5 presents the beam characteristics at the center of the undulator for the total beam, the $176 \pm 0.1 \mathrm{MeV}$ and $176 \pm 0.5 \mathrm{MeV}$ slices. Three cases are considered: using the distribution of Figure 5b without the slit, with the slit opened at $3.6 \mathrm{~mm}$ and using a flat-top beam of $176 \pm 5 \%$ $\mathrm{MeV}$ with the slit opened at $3.6 \mathrm{~mm}$. In the flat-top case of $\sigma_{\gamma}=5 \%$ with the slit opened 

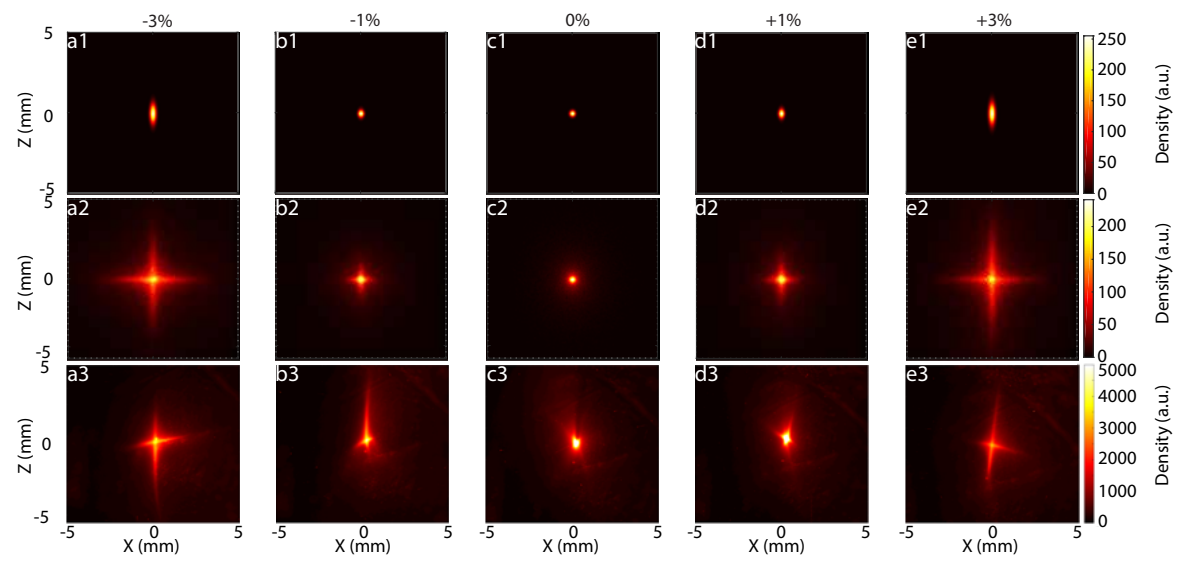

Figure 7. Electron beam transverse shape simulated for the baseline parameters (a1e1), for the average beam distribution (a2-e2) with the "undulator entrance" optics and measurement (a3-e3) at the undulator entrance for the relative gradient change of QUAPEVA 2 of $+3 \%$ (a), $+1 \%$ (b), $0 \%$ (c), $-1 \%$ (d) and $-3 \%$ (e). Simulation case with the average beam distribution Figure $5 \mathrm{c}, \epsilon_{x, i}, \epsilon_{z, i}=0.2 \mathrm{~mm} . \mathrm{mrad}, \sigma_{z, i}^{\prime}=2 \mathrm{mrad}$, $\sigma_{x, i}^{\prime}=3.12 \mathrm{mrad}$.

at $3.6 \mathrm{~mm}$, the brightness (Equation (2)) of $5.53 \times 10^{4} \mathrm{~A} /\left(\mathrm{mm}^{2} \mathrm{mrad}^{2}\right)$ for the on axis beam is significantly reduced to $3.76 \times 10^{4} \mathrm{~A} /\left(\mathrm{mm}^{2} \mathrm{mrad}^{2}\right)$ with the $2 \mathrm{mrad}$ pointing error. For the beam distribution of Figure $5 \mathrm{~b}$ without slit in the case without pointing, from an initial total charge of $100 \mathrm{pC}$ only $28 \mathrm{pC}$ arrives at the center of the undulator. With a $2 \mathrm{mrad}$ vertical pointing error angle with respect to the axis, the charge at the undulator center is reduced to $26 \mathrm{pC}$. The charge of the $176 \pm 0.5 \mathrm{MeV}$ slice is mostly conserved. The increase of $\sigma_{\gamma}$ makes the chromatic term (Equation (1)) larger, which results in $\epsilon_{x}$ and $\epsilon_{z}$ at the undulator center doubling their initial value. $\sigma_{s}$ is increased by a factor 1.4. When a 2 mrad vertical pointing is introduced for the total beam (slice $176 \pm 0.5 \mathrm{MeV})$ case, the $\epsilon_{z}$ at the center of the undulator increases, which translates into a decrease of the brightness of a factor 1.07 (1.13). When the $3.6 \mathrm{~mm}$ slit is introduced at the center of the chicane, the charge of the total beam arriving at the center of the undulator is reduced to $10.6 \mathrm{pC}$ and $\epsilon_{z}$ drops by a factor 8 for the on-axis beam. However, the $176 \pm 0.5 \mathrm{MeV}$ and $176 \pm 0.1 \mathrm{MeV}$ slices charge barely changes, i.e. the designed transport line with slit indeed cuts mostly energies outside the range of interest $176 \pm 0.5 \mathrm{MeV}$. The slice brightness of the Figure $5 \mathrm{~b}$ distribution with the 3.6 $\mathrm{mm}$ slit is one order of magnitude lower than in the flat-top case evidencing how much the $\sigma_{\gamma}$ affects the beam quality. In both cases using the $3.6 \mathrm{~mm}$ slit, a degradation of the beam due to pointing is confirmed, even for the slice $176 \pm 0.1 \mathrm{MeV}$.

To tackle the shot-to-shot pointing, a beam pointing compensation method (BPAC) has been put in place. In such a method, the response matrix of the line linking the position and dispersion of the electron beam to the transverse offset of the QUAPEVAs is numerically calculated and then applied to the transverse offsets of the QUAPEVA to correct orbit dispersion [37]. 


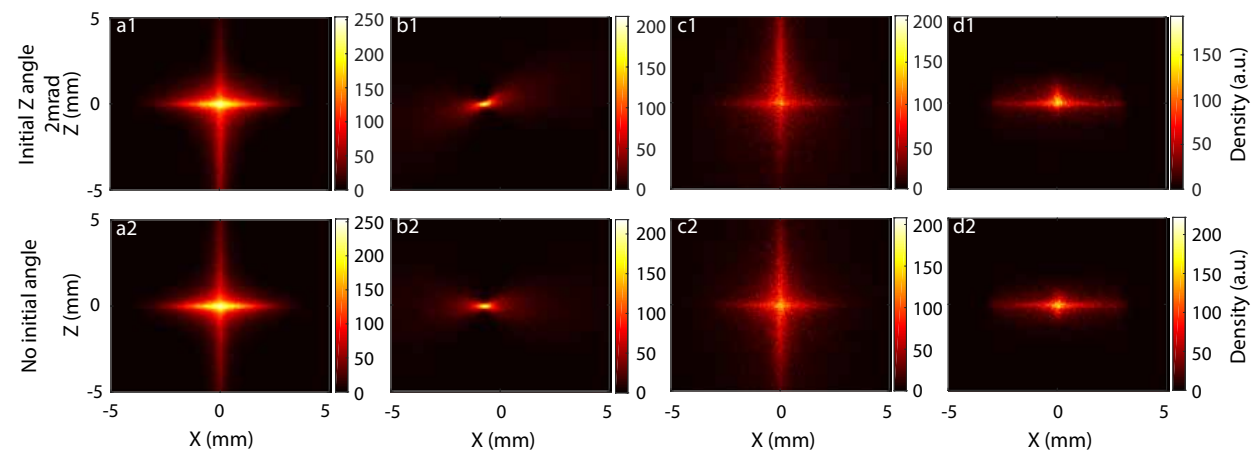

Figure 8. Simulated transverse beam shape along the transport line on screens located after QUAPEVAS (a1-2), in the middle of the chicane (b1-2), undulator entrance (c12 ) and undulator exit (d1-2), for an on axis beam (a-d2) and for a beam with $2 \mathrm{mrad}$ vertical pointing (a-d1), transported with the "slit undulator" optics with the average beam (Figure 5a), $\epsilon_{x, i}, \epsilon_{z, i}=0.2 \mathrm{~mm} . \mathrm{mrad}, \sigma_{z, i}^{\prime}=2 \mathrm{mrad}, \sigma_{x, i}^{\prime}=3.12 \mathrm{mrad}$.

Table 5. RMS Beam length $\sigma_{s}$, charge $Q$, RMS $\epsilon_{x}$, RMS $\epsilon_{y}$ and brigthness $B$ (Equation (2)) at the undulator center in the case of the "undulator slit" optics and average beam of Figure 5b, without and with slit of $3.6 \mathrm{~mm}$ aperture for the total beam, for the $176 \pm 0.5 \mathrm{MeV}$ and $176 \pm 0.1 \mathrm{MeV}$ slices and for the case of a flat-top beam of $176 \pm 5 \% \mathrm{MeV}$, with $\epsilon_{x, i}, \epsilon_{z, i}=0.2 \mathrm{~mm} . \mathrm{mrad}, \sigma_{z, i}^{\prime}=2 \mathrm{mrad}, Q_{i}=100 \mathrm{pC}, \sigma_{x, i}^{\prime}=3.12$ mrad.

\begin{tabular}{|c|c|c|c|c|c|c|c|c|}
\hline Beam distribution & Beam part & $\begin{array}{c}\text { Slit } \\
(\mathrm{mm})\end{array}$ & $\begin{array}{l}\text { Pointing } \\
(\text { mrad })\end{array}$ & $\begin{array}{c}\sigma_{s} \\
(\mu m)\end{array}$ & $\begin{array}{l}Q \\
(p C)\end{array}$ & $\begin{array}{c}\epsilon_{x} \\
(m m \text { mrad })\end{array}$ & $\begin{array}{l}\epsilon_{z} \\
(m m \text { mrad })\end{array}$ & $\begin{array}{c}B \\
\left(\frac{A}{m m^{2} m r a d^{2}}\right)\end{array}$ \\
\hline \multirow[t]{2}{*}{ Figure $5 b$} & Total beam & - & 0 & 0.40 & 28.4 & 112.4 & 454.3 & 9.86 \\
\hline & & & 2 & 0.38 & 26.6 & 112.1 & 489.2 & 9.051 \\
\hline \multirow[t]{2}{*}{ Figure 5b } & $176 \pm 0.5 \mathrm{MeV}$ & - & 0 & 0.013 & 0.5 & 9.2 & 3.1 & $0.92 \times 10^{3}$ \\
\hline & & & 2 & 0.014 & 0.48 & 9.2 & 3.5 & $0.74 \times 10^{3}$ \\
\hline \multirow[t]{2}{*}{ Figure $5 b$} & $176 \pm 0.1 \mathrm{MeV}$ & - & 0 & 0.013 & 0.043 & 1.2 & 0.4 & $5 \times 10^{4}$ \\
\hline & & & 2 & 0.012 & 0.043 & 1 & 0.5 & $5.53 \times 10^{4}$ \\
\hline \multirow[t]{2}{*}{ Figure 5b } & Total beam & 3.6 & 0 & 0.14 & 10.56 & 117.4 & 56 & 79.1 \\
\hline & & & 2 & 0.14 & 10.49 & 117.6 & 73.2 & 60.38 \\
\hline \multirow[t]{2}{*}{ Figure $5 b$} & $176 \pm 0.5 \mathrm{MeV}$ & 3.6 & 0 & 0.014 & 0.48 & 9.2 & 3.1 & $0.89 \times 10^{3}$ \\
\hline & & & 2 & 0.014 & 0.48 & 9.1 & 3.6 & $0.74 \times 10^{3}$ \\
\hline \multirow[t]{2}{*}{ Figure 5b } & $176 \pm 0.1 \mathrm{MeV}$ & 3.6 & 0 & 0.010 & 0.046 & 0.9 & 0.4 & $8.74 \times 10^{4}$ \\
\hline & & & 2 & 0.012 & 0.04 & 1.0 & 0.5 & $4.16 \times 10^{4}$ \\
\hline \multirow[t]{2}{*}{ Flat-top beam } & $176 \pm 0.5 \mathrm{MeV}$ & 3.6 & 0 & 0.01 & 0.48 & 4.6 & 1.4 & $5.53 \times 10^{4}$ \\
\hline & & & 2 & 0.01 & 0.48 & 4.6 & 2 & $3.76 \times 10^{4}$ \\
\hline \multirow[t]{2}{*}{ Flat-top beam } & $176 \pm 0.1 \mathrm{MeV}$ & 3.6 & 0 & 0.005 & 0.046 & 0.4 & 0.2 & $6.24 \times 10^{5}$ \\
\hline & & & 2 & 0.006 & 0.040 & 0.4 & 0.3 & $4.26 \times 10^{5}$ \\
\hline
\end{tabular}

\subsection{Sensitivity to magnet error}

Following the proportionality in Equation (1) [26], a change in the tilt converts into an equal effect on the emittance. The roll angle of the quadrupole magnet $\theta$, due to its inherent imperfections, is defined as $\theta=-\frac{1}{2} \arctan \left(\frac{a_{2}}{b_{2}}\right)$, with $b_{2}$ the normal quadrupolar term and $a_{2}$ the skew quadrupolar term. Figure 9 compares simulations 

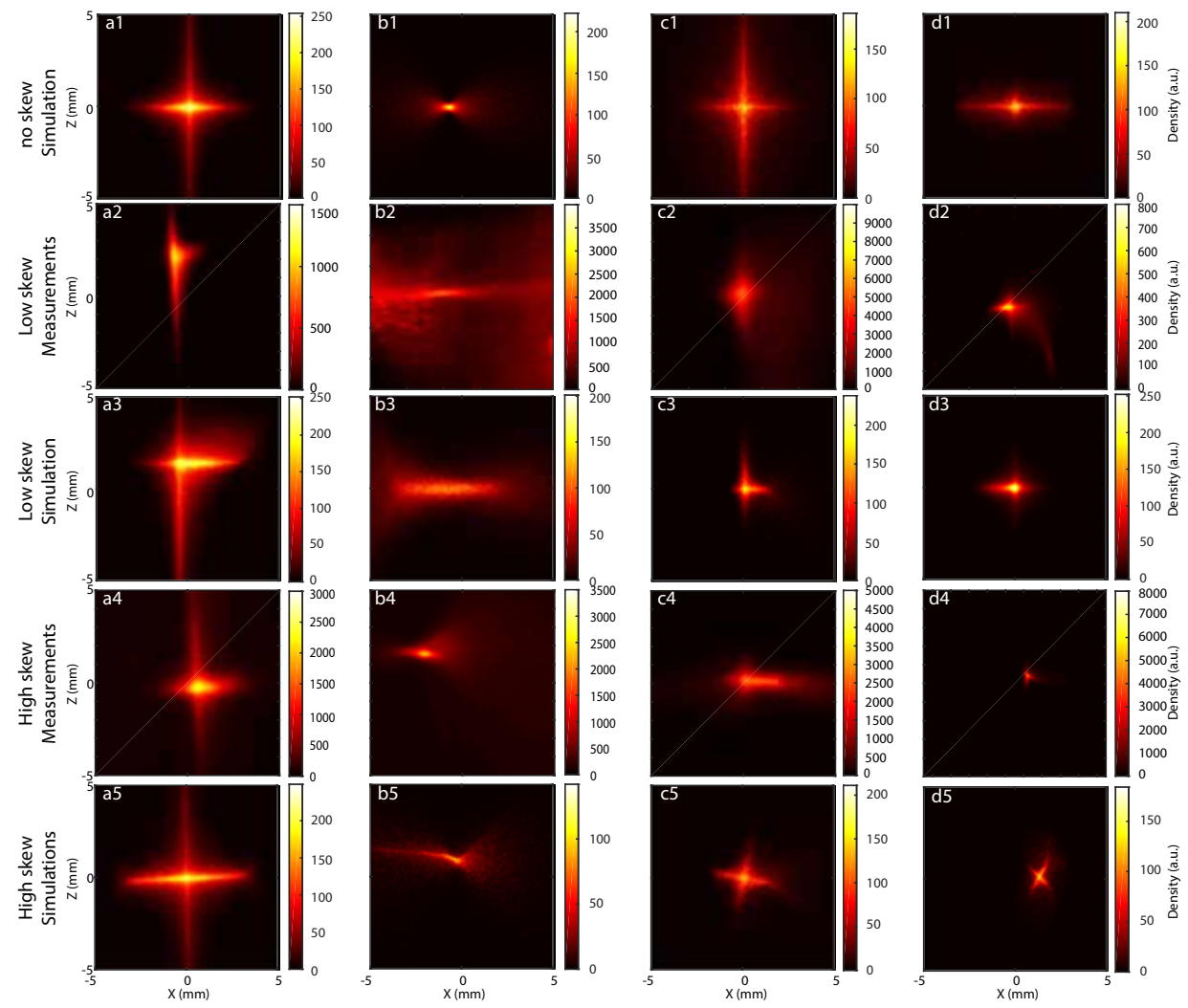

Figure 9. Transverse beam shape along the transport line at screen after QUAPEVAS (a1-5), in the middle of the chicane (b1-5), undulator entrance (c1-5) and undulator exit (d1-5) measured (a-d2, a-d4) and simulated (a-d1, a-d3, a-d5), with no (a-d1), low (a-d2-3) and high (a-d4-5) roll angle, the optics "undulator slit" for (a1-3) and (b-d1), "slit" for (a4-5) and (b2-5), "undulator entrance" (c2-3), "undulator center" (c4-5) and (d4-5), "undulator exit" (d2-3) for the average beam (Figure 5b) (a-d1, a-d3) and single-shot (Figure 5d) (a-d5). Parameters: $\epsilon_{x, i}, \epsilon_{z, i}=0.2 \mathrm{~mm}$.mrad, $\sigma_{z, i}^{\prime}=2$ $\operatorname{mrad}, \sigma_{x, i}^{\prime}=3.12 \mathrm{mrad}$ for (a-d1-3) and $\sigma_{x, i}^{\prime}=4.7 \mathrm{mrad}$ for (a-d4-5). QUAPEVAs High roll angles: $3.3 \mathrm{mrad},-9 \mathrm{mrad},-9.4 \mathrm{mrad}$. Low roll angles: $-0.3 \mathrm{mrad}, 0.7 \mathrm{mrad}$, 0.05 mrad.

and measurements of the transverse beam shape at the four screens along the line for different $\theta$ values. Measured QUAPEVA high and low $\theta$ correspond to different shimming configurations [26]. Figure 9a1-d1 presents the transport simulation with "undulator slit" optics without roll angle and the initial beam distribution of Figure 5b. The beam is well focused vertically at the slit to achieve an accurate electron energy selection and in both directions at the center of the undulator, while at the undulator entrance and exit the different focus for the electron energies leads to a cross shape. In the low roll angle case, measurements (Figure 9a-d2) are well reproduced by simulations (Figure 9a-d3). Table 6 compares the beam RMS horizontal and vertical sizes found in simulation and experiment (Figure 9a,c,d2-3). The difference can be easily caused by the fluctuations of the beam distribution. In the presence of the high roll angle, the beam suffers from a tilt that causes dispersion, affects the emittance (Equation (1)) and 
Table 6. Simulated and measured transverse beam shape (Figure 9a,c,d2-3) RMS horizontal $\left(\sigma_{x}\right)$ and vertical $\left(\sigma_{z}\right)$ sizes for the low roll angle case.

\begin{tabular}{cccccc}
\hline & & \multicolumn{2}{c}{ Simulation } & \multicolumn{2}{c}{ Measurement } \\
\hline \multicolumn{1}{c}{ Figures } & Position & $\sigma_{x}$ & $\sigma_{z}$ & $\sigma_{x}$ & $\sigma_{z}$ \\
\hline & & $m m$ & $m m$ & $m m$ & $m m$ \\
\hline Figure 9a2-3 & After QUAPEVAs & 1.16 & 3.11 & 1.49 & 0.86 \\
Figure 9c2-3 & Undulator entrance & 1.16 & 1.19 & 1.03 & 1.58 \\
Figure 9d2-3 & Undulator exit & 0.75 & 0.69 & 1.29 & 0.93 \\
\hline
\end{tabular}

degrades the transport [26]. The simulation is able to well reproduce the shape of the beam along the line (Figure 9a-d4-5).

\section{Conclusion}

The limitations of LPA sources still pose a setback on the quality of the produced electron beam. Thus, a specific transport line is crucial to control the phase space of the electron beam in order to transport a undulator like photon source for applications. In the COXINEL case, the beam measured after the source has a much higher energy spread and divergence with a lower charge density than the expected reference parameters. The transverse beam shape along the line varies with the triplet gradient setting due to chromatic effects, leading to the appearance of a cross shape due to the different position of vertical and horizontal focusing for the different energies. The beam size is substantially increased even for an energy spread of $1 \%$ beam centered on the energy of interest $176 \mathrm{MeV}$. Pointing variations of the laser leads to an emittance growth and additional electron beam losses, due to the line aperture, and further brightness reduction. Finally, the existence of a roll angle in the QUAPEVA triplet causes an observable tilt of the electron beam along the line and increases the emittance.

Funding: This work was partially supported by the European Research Council for the Advanced Grants COXINEL (340015, PI: M.-E. C.) and X-Five (339128, PI: V.M.), EuPRAXIA design study (6538782), the Fondation de la Cooperation scientifique (QUAPEVA-2012-058T), the Agence Nationale de la Recherche [projet Blanc DYNACO 2010-042301], the Agence Nationale de la Recherche through the LABEX CEMPI (ANR-11-LABX-0007), as well as by the Ministry of Higher Education and Research, Hauts de France council and European Regional Development Fund (ERDF) through the Contrat de Projets Etat-Region (CPER Photonics for Society P4S).

Acknowledgments: The authors acknowledge SOLEIL staff for their support and 
in particular the Magnestism and Insertion device group and J. L. Lancelot, F. Forest, O. Cosson at Sigmaphi for the joint development of the QUAPEVA magnets. 
[1] Tajima T, Dawson JM. Laser electron accelerator. Physical Review Letters. 1979;43(4):267.

[2] Esarey E, Schroeder C, Leemans W. Physics of laser-driven plasma-based electron accelerators. Reviews of Modern Physics. 2009;81(3):1229.

[3] Leemans W, Gonsalves A, Mao HS, Nakamura K, Benedetti C, Schroeder C, et al. Multi-GeV electron beams from capillary-discharge-guided subpetawatt laser pulses in the self-trapping regime. Physical review letters. 2014;113(24):245002.

[4] Wang X, et al. Quasi-monoenergetic laser-plasma acceleration of electrons to $2 \mathrm{GeV}$. Nature communications. 2013;4:1988.

[5] Lundh O, Lim J. O. Lundh, J. Lim, C. Rechatin, L. Ammoura, A. Ben-Ismail, X. Davoine, G. Gallot, J.-P. Goddet, E. Lefebvre, V. Malka, and J. Faure, Nat. Phys. 7, 219 (2011). Nat Phys. 2011;7:219.

[6] Rechatin C, Faure J, Ben-Ismail A, Lim J, Fitour R, Specka A, et al. Controlling the phasespace volume of injected electrons in a laser-plasma accelerator. Physical review letters. 2009;102(16):164801.

[7] Ting A, Esarey E, Sprangle P. Nonlinear wake-field generation and relativistic focusing of intense laser pulses in plasmas. Physics of Fluids B: Plasma Physics. 1990;2(6):1390-1394.

[8] Feit M, Komashko A, Musher S, Rubenchik A, Turitsyn S. Electron cavitation and relativistic self-focusing in underdense plasma. Physical Review E. 1998;57(6):7122.

[9] Esarey E, Hubbard R, Leemans W, Ting A, Sprangle P. Electron injection into plasma wakefields by colliding laser pulses. Physical Review Letters. 1997;79(14):2682.

[10] Faure J, Rechatin C, Norlin A, Lifschitz A, Glinec Y, Malka V. Controlled injection and acceleration of electrons in plasma wakefields by colliding laser pulses. Nature. 2006;444(7120):737.

[11] Bulanov S, Naumova N, Pegoraro F, Sakai J. Particle injection into the wave acceleration phase due to nonlinear wake wave breaking. Physical Review E. 1998;58(5):R5257.

[12] Audet T, Hansson M, Lee P, Desforges F, Maynard G, Dobosz Dufrénoy S, et al. Investigation of ionization-induced electron injection in a wakefield driven by laser inside a gas cell. Physics of Plasmas. 2016;23(2):023110.

[13] Osterhoff J, Popp A, Major Z, Marx B, Rowlands-Rees T, Fuchs M, et al. Generation of stable, low-divergence electron beams by laser-wakefield acceleration in a steady-state-flow gas cell. Physical review letters. 2008;101(8):085002.

[14] McGuffey C, Thomas A, Schumaker W, Matsuoka T, Chvykov V, Dollar F, et al. Ionization induced trapping in a laser wakefield accelerator. Physical Review Letters. 2010;104(2):025004.

[15] Schroeder C, Esarey E, Geddes C, Benedetti C, Leemans W. Physics considerations for laser-plasma linear colliders. Physical Review Special Topics-Accelerators and Beams. 2010;13(10):101301.

[16] Molodozhentsev A, Pribyl L. ELI Eectron Beam Line for Laser-plasma-driven Undulator X-ray Source. Proceedings of IPAC2016, Busan, Korea. 2016:4005-4007.

[17] Madey JM. Stimulated emission of bremsstrahlung in a periodic magnetic field. Journal of Applied Physics. 1971;42(5):1906-1913.

[18] Kondratenko A, Saldin E. Generating of coherent radiation by a relativistic electron beam in an ondulator. Part Accel. 1980;10:207-216.

[19] Huang Z, Kim KJ. Review of x-ray free-electron laser theory. Physical Review Special TopicsAccelerators and Beams. 2007;10(3):034801.

[20] Couprie ME. New generation of light sources: Present and future. Journal of Electron Spectroscopy and Related Phenomena. 2014;196:3-13.

[21] Grüner F, et al. Design considerations for table-top, laser-based VUV and X-ray free electron lasers. Applied Physics B. 2007;86(3):431-435.

[22] Nakajima K. Compact X-ray sources: Towards a table-top free-electron laser. Nature physics. 2008;4(2):92-93.

[23] Floettmann K. Some basic features of the beam emittance. Physical Review Special Topics- 
Accelerators and Beams. 2003;6(3):034202.

[24] Antici P, et al. Laser-driven electron beamlines generated by coupling laser-plasma sources with conventional transport systems. J Appl Phys. 2012;112(4):044902.

[25] Migliorati M, et al. Intrinsic normalized emittance growth in laser-driven electron accelerators. Phys Rev ST Accel Beams. 2013;16(1):011302.

[26] Oumbarek Espinos D, Ghaith A, André T, Kitégi C, Sebdaoui M, Loulergue A, et al. Skew Quadrupole Effect of Laser Plasma Electron Beam Transport. Applied Sciences. 2019;9(12):2447.

[27] Mostacci A, Bellaveglia M, Chiadroni E, Cianchi A, Ferrario M, Filippetto D, et al. Chromatic effects in quadrupole scan emittance measurements. Physical Review Special TopicsAccelerators and Beams. 2012;15(8):082802.

[28] Lejeune C, Aubert J. Emittance and brightness: Definitions and measurements. Applied Charged Particle Optics. 1980;(Supp. 13A).

[29] Dattoli G, Renieri A, Torre A. Lectures on the Free Electron Laser Theory and Related Topics. 1993;.

[30] Bonifacio R. R. Bonifacio, C. Pellegrini, and LM Narducci, Opt. Commun. 50, 373 (1984). Opt Commun. 1984;50:373.

[31] Liu T, Zhang T, Wang D, Huang Z. Compact beam transport system for free-electron lasers driven by a laser plasma accelerator. Physical Review Accelerators and Beams. 2017;20(2):020701.

[32] van Tilborg J, Barber S, Isono F, Schroeder C, Esarey E, Leemans W. Free-electron lasers driven by laser plasma accelerators. In: AIP Conference Proceedings. vol. 1812. AIP Publishing; 2017. p. 020002 .

[33] Leemans W, Esarey E. Laser-driven plasma-wave electron accelerators. Phys Today. 2009;62(3):44-49.

[34] Khojoyan M, et al. Transport studies of LPA electron beam towards the FEL amplification at COXINEL. Nucl Instr Meth Phys Res A. 2016;829:260-264.

[35] Loulergue A, et al. COXINEL baseline reference case LUNEX5. COXINEL Report. 2015;

[36] Benabderrahmane C, et al. Development and operation of a $\operatorname{Pr} 2$ Fe 14 B based cryogenic permanent magnet undulator for a high spatial resolution x-ray beam line. Physical Review Accelerators and Beams. 2017;20(3):033201.

[37] André T, et al. Control of laser plasma accelerated electrons for light sources. Nature Communications. 2018;9(1):1334.

[38] Couprie M, Labat M, Evain C, Marteau F, Briquez F, Khojoyan M, et al. An application of laserplasma acceleration: towards a free-electron laser amplification. Plasma Physics and Controlled Fusion. 2016;58(3):034020.

[39] Labat M, Loulergue A, Andre T, Andriyash I, Ghaith A, Khojoyan M, et al. Robustness of a plasma acceleration based free electron laser. Physical Review Accelerators and Beams. 2018;21(11):114802.

[40] André T, Andriyash I, Basset C, Benabderrahmane C, Berteaud P, Bielawski S, et al. First electron beam measurements on coxinel. In: 7th International Particle Accelerator Conference (IPAC'16), Busan, Korea; 2016. p. 712-715.

[41] Marteau F, Ghaith A, N'Gotta P, Benabderrahmane C, Valléau M, Kitegi C, et al. Variable high gradient permanent magnet quadrupole (QUAPEVA). Applied Physics Letters. 2017;111(25):253503.

[42] Ghaith A, Kitegi C, André T, Valléau M, Marteau F, Vétéran J, et al. Tunable high gradient quadrupoles for a laser plasma acceleration based FEL. Nuclear Instruments and Methods in Physics Research Section A: Accelerators, Spectrometers, Detectors and Associated Equipment. 2018;909:290-293.

[43] Mihara T. A Super Strong Permanent Magnet Quadrupole for the Final Focus in a Linear Collider. SLAC National Accelerator Lab., Menlo Park, CA (United States); 2018.

[44] Ghaith A, Kitegi C, André T, Valléau M, Marteau F, Vétéran J, et al. Tunable high gradient 
quadrupoles for a laser plasma acceleration based FEL. Nuclear Instruments and Methods in Physics Research Section A: Accelerators, Spectrometers, Detectors and Associated Equipment. 2018;909:290-293.

[45] Maier A, Meseck A, Reiche S, Schroeder C, Seggebrock T, Gruener F. Demonstration scheme for a laser-plasma-driven free-electron laser. Physical Review X. 2012;2(3):031019.

[46] Couprie ME, Loulergue A, Labat M, Lehe R, Malka V. Towards a free electron laser based on laser plasma accelerators. J Phys B. 2014;47(23):234001.

[47] Loulergue A, et al. Beam manipulation for compact laser wakefield accelerator based free-electron lasers. New Journal of Physics. 2015;17(2):023028.

[48] Payet J, et al. BETA code. CEA, SACLAY;.

[49] Flöttmann K, Astra A. A space charge tracking algorithm. ASTRA), http://www desy de/ mpyflo/Astra_dokumentation. 2007; .

[50] Borland M. Elegant: A flexible SDDS-compliant code for accelerator simulation. Argonne National Lab., IL (US); 2000.

[51] Agapov I, Geloni G, Tomin S, Zagorodnov I. OCELOT: A software framework for synchrotron light source and FEL studies. Nuclear Instruments and Methods in Physics Research Section A: Accelerators, Spectrometers, Detectors and Associated Equipment. 2014;768:151-156. 